1

\title{
An ectosymbiosis-based mechanism of eukaryogenesis
}

3

4

5

\section{Abstract}

(10)
diversity of sequence motifs in eukaryotic Hsp70s rendered invalid the endosymbiosis-first theory that mitosome- and hydrogenosome-containing amitochondriate eukaryotes (AMIs) arose from mitochondriate eukaryotes (MTEs) via reductive evolution. Instead, evidence of widespread ectosymbioses indicated that eukaryogenesis was started by an archaeal parent via its acquisition of archaeal proteins through 'accelerated gene adoption', and bacterial proteins from ectosymbionts including a clostridial ectosymbiont that supplied its [Fe] hydrogenase and pyruvate:ferredoxin oxidoreductase genes to the AMIs. Subsequent endosymbiosis with Tistrella gave rise to mitochondria with the participation of other alphaproteobacteria. The high frequencies of top similarity bitscores displayed by Giardia, Edhazardia and Trichomonas toward Aciduliprofundum boonei (Abo) pertaining to the enzymes of DNA biology, far surpassing the frequencies toward any Asgard or TACK archaeon, established Abo as the source of these enzymes in eukaryotes, and the archaeal parent of Eukarya.

For over a century endosymbiosis has been regarded as the basis for eukaryogenesis, with recent focus placed on an archaeal parent-alphaproteobacterial endosymbiosis ${ }^{1}$. It was pointed out based on the SSU rRNA sequence of Vairimorpha necatrix that Microsporidia branched very early from the other eukaryotes on the SSU rRNA tree ${ }^{2}$. The evolution of MTEs from the AMIs was postulated by the 'Archezoa' theory based on the autogenous evolution of a primitive phagotrophic archezoan ${ }^{3}$, and by the 'crown' theory based on the locations of MTE branches above the AMI branches on the SSU rRNA tree ${ }^{4}$. However, the discoveries of typical mitochondrial proteins in the AMIs gave rise to the 'endosymbiosis first' theory that the presence of mitochondrial proteins in AMIs implies the harboring of mitochondria or their endosymbiotic ancestors by the AMIs in the past ${ }^{5,6}$. As a result, the proposal of Lokiarchaeota being a suitable archaeal parent was held up by the missing mechanism for importing an alphaproteobacterial endosymbiont ${ }^{7}$, and the proposal of the Aciduliprofundum archaeons being appropriate archaeal parents would not be implementable if these small archaeons are required to accommodate an upfront endosymbiont ${ }^{8}$. In addition, searches have not yielded a consensual phylogenetic location for the Last Eukaryotic Common Ancestor (LECA) ${ }^{9-12}$, and autogenous development of eukaryotes has not given a better fit to omics data than endosymbiosis $^{13}$. In view of these difficulties, the present study has been directed to an investigation of the possibility of LECA formation through ectosymbiosis instead of endosymbiosis. 
43

44

45

Rejection of the primitivity of AMIs despite their low branchings on SSU rRNA trees stemmed from the supposition that their long branches may be due to an accelerated rate of evolution, making them susceptible to the artifact of long branch attraction ${ }^{14}$. However, the tree in Figure 1, built using the maximum likelihood method that minimized long branch attraction artifacts $^{15}$, not only positioned the AMIs as low branchers, but also grouped the Microsporidia species into a clade.

The branches of the Amoebozoa AMI species Ein and Enh (see Table 1 for abbreviations of species names), which reductively evolved from Amoebozoa MTE species ${ }^{16}$, were indeed much longer than the MTE branches of Asu, Ddi and Dpu. However, despite their long branches, Ein and Enh were included in the same Amoebozoa clade as Asu, Ddi and Dpu on the tree. Both Mon which lost the mitochondria or mitochondria-related organelles (MROs) it once possessed ${ }^{17}$, and $\mathrm{Cpa}$ which has degenerate mitochondria ${ }^{18}$ also did not move to lowbranching positions on the tree on account of their experience of reductive evolution. The nucleomorphs with their vestigial genomes likewise stayed with their ancestral red or green algae on the tree ${ }^{19}$. Accordingly, there was little basis to assume that the clade of nine AMI Microsporidia species had moved to a low branching position on the tree because of their reductive evolution from fungi ${ }^{20}$, away from the modern fungal Sce, Spo, Lbi, Ncr, Mci, Bde and Spu sitting at the top of the tree. Among the Microsporidia, Ecu with its $2.9 \mathrm{Mb}$ genome was much more reduced in size compared to some of the other microsporidians ${ }^{21}$, but it was located within the same clade as other microsporidians on either the SSU rRNA tree (Figure 1) or a tree for 53 concatenated proteins ${ }^{22}$. Therefore, the evidence for Microsporidia being an independently evolved AMI group rather than products of reductive evolution, in contradiction to any theory that requires the derivation of all AMIs from MTEs, was strong.

The motifs of eukaryotic Hsp70 sequences also rendered uncertain the evidence for the endosymbiosis-first theory founded on the affinities between mitochondrial proteins and their counterparts in the AMIs ${ }^{23}$. Figure 2 shows the Group-I EKP/DKP/GKP and Group-II NGDAWV motifs, which were both absent from the archaeal sequences of Abo, Lok and Hgi but resembled bacterial sequences: the EKP-motif resembled that of Aba, the DKP-motif resembled those of Cur and Kae, and the GKP-motif resembled those of Mca and Eco. The NGDAWV-motif was found in not only the alphaproteobacteria Tis, Atu and Rru, but also the gammaproteobacterium Eco and the deltaproteobacterium Pel. Such diversified motifs in both AMIs and MTEs were in keeping with the profuse variety of prokaryotic proteins found in the proteomes of eukaryotes ${ }^{8,24-27}$, but neither the diversity nor the apparent paucity or lack of the EKP motif in the MTEs was consistent with the origin of all MTE and AMI Hsp70s from a single alphaproteobacterial endosymbiont.

\section{Ectosymbiotic mechanism of eukaryogenesis}

In view of the unlikelihood of endosymbiosis-first, and the intrinsic compatibility of ectosymbiosis with AMI primitivity and Hsp70 diversity, an ectosymbiosis-based mechanism of eukaryogenesis was examined in Figure 3.

In Stage 1 of this mechanism, bacterial ectosymbionts were attached to an archaeal parent. Enwrapment of part or whole of the ectosymbionts by the archaeal parent led to the influx of bacterial genes, proteins and metabolic products, whereupon bacterial ester-lipids 
85

86

87

88

89

90

91

92

93

94

95

96

97

98

99

100

101

102

103

104

105

106

107

108

109

110

111

112

113

114

115

116

117

118

119

120

121

122

123

124

125

126

127

128

129

(blue) infiltrated the archaeal ether-lipid (red) membranes to form initially chimeric and later fully bacterial membranes. The archaeal host and the ectosymbionts worked together in Stage 2 to develop the mitosome, which contributed protein presequences for organellar targeting ${ }^{28}$ and took part in $\mathrm{Fe}-\mathrm{S}$ cluster assembly ${ }^{29}$. Upon departure of the ectosymbionts from the archaeal host, a mitosome-containing LECA/MSO emerged. It was suggested that an early membrane-curving module might play a role in the origin of nuclear pore complex and coated vesicles $^{30}$, in which case extra curvable prokaryotic membranes such as those of $\mathrm{Abo}^{31}$ would merit extensive investigation. One of the incentives for the formation of cell nucleus might be that archaeal introns were spliced by an endoribonuclease at RNA bulge-helix-bulge motifs. Without a nucleus, some mRNAs from bacterial ectosymbionts would risk misrecognition as introns by the archaeal enzyme and erroneously excised.

At Stage 3 the archaeal host collaborated further with the ectosymbionts to replace the mitosome with hydrogenosome, and generated hydrogenosome-containing AMIs. The bioenergetic resourcefulness of the hydrogenosome was illustrated by the amitochondriate hydrogenosome-containing animal phylum Loricifera ${ }^{32}$. Further archaeal-bacterial cooperation produced alphaproteobacterial endosymbionts at Stage 4, which led to the formation of mitochondria and the MTEs. Thus the ectosymbiotic mechanism gives rise to an LECA that is AMI in nature at Stage 2, and the many ectosymbionts participating in Stages 14 , far exceeding the ones illustrated in Figure 3, would readily account for the multiple bacteriaderived Hsp70 motifs in eukaryotes in Figure 2.

\section{Prokaryotic proteins in eukaryotic proteomes}

The eukaryotic proteomes were enriched with prokaryote-derived proteins. The bacterial/archaeal protein ratios of $0.73 / 0.85 / 1.09 / 1.26 / 1.32$ for Eae/Gla/Trv/Sce/Dme respectively pointed to the diminishing proportion of archaeal proteins as evolution of the MTEs proceeded, possibly due to the greater functional scope offered by bacterial proteins relative to archaeal proteins. The proteomes of Per, Bbo, Pfa and Bho were more primitive in character than those of other MTEs based on their higher proportions of archaeal proteins (Figure 4).

The entry of prokaryotic proteins into eukaryotes could be mediated by a number of different mechanisms. While horizontal gene transfers (HGTs) might be limited by low rates of acceptance by the gene recipients ${ }^{33}$, it was found recently that some archaea displayed an 'accelerated gene adoption' (AGA) phenotype detectible by the elevated levels of bacteriaderived proteins in their proteomes ${ }^{8}$. Moreover, the genes of the bacterial species Rpr, Bap, Ctr, Mpn, and Tpa furnished relatively few protein coding genes that were adopted by the AGAactive archaeons, and their proteins were also depleted in the proteomes of both Gla and Trv, demonstrating the key role played by AGA in controlling influx of bacterial genes into the eukaryogenic lineage. Also the paucity of AGA activity in bacteria would also render them much less effective than archaea in hosting eukaryogenesis owing to the importance of collection of exogenous genes by the host in this regard. Anaerobic Abo and its facultatively anaerobic companion species Acf, on account of their top average similarity bitscores among archaeons toward Gla and Trv proteomes and the PEP-utilizing enzyme mobile domain of Gla, along with their outstanding AGA activity, emerged as leading candidate archaeal parents ${ }^{8}$. When the DNA-biology enzymes/proteins belonging to the Cluster of Ortholog Groups (COG) for replication, recombination and repair $^{34}$ were analyzed with respect to the similarity bitscores displayed by primitive AMIs toward Abo and various Asgard and TACK archaeons 
which included some attractive candidate archaeal parents ${ }^{8,35}$,the frequencies of top similarity bitscores received by Abo from the primitive AMIs pertaining to the DNA-biology enzymes, viz. for 8/21 enzymes from Gla, 6/20 from Eae, 7/29 from Trv and 7/20 from Gin, far surpassed those of any Asgard or TACK archaeon tested (Figure 5a and Supplementary Figure S1). These results identified Abo as the major source of the DNA-biology enzymes in the genomes of all of these primitive AMIs, and therefore Abo as the archaeal parent of Eukarya.

It has been pointed out that, because different groups of present-day eukaryotes share few common features, the vertical inheritance between them remains an assumption ${ }^{35}$. However, Figure 5b panels 1-8 show that a series of archaeal proteins including DNA-biology enzymes and eukaryotic signature proteins ${ }^{36}$ (ESPs) maintained fairly steady similarity bitscores toward both AMIs and MTEs ranging from Microsporidia to Hsa, which attested to the vertical transmission of these proteins between eukaryotes, and their significance as part of the core of eukaryotic proteomes. Bho and its subtypes Bs1, Bs4, and Bs3 (no reported proteome and therefore not included in Figure 4), which were in possession of both hydrogenosome components and partial mitochondria ${ }^{37}$, shared with the mitosome- and hydrogenosome-containing AMIs as well as the MTEs Bbo and Pfa the presence of Ssp-TteHth proteins in their proteomes, thus pointing to a relationship between these firmicute species and the MRO-containing AMIs in keeping with the metabolic resemblance between hydrogenosome and Clostridium ${ }^{38}$. This was confirmed in Figure $5 \mathrm{~b}$ panels 9-10, where the MRO-containing AMIs other than Microsporidia exhibited substantial bitscores toward the [Fe] hydrogenase and pyruvate:ferredoxin oxidoreductase (PFO) of clostridial Tte. The low or zero bitscores exhibited by the Microsporidia could be the result of similarity erosion due to the extensive mutation of microsporidial proteins: Nbo was the only microsporidian in the 9member clade on the SSU rRNA tree with any residual bitscore toward the PFO of Tte.

Previously, Paracoccus was found to branch near eukaryotes on the trees for the subunits of succinate dehydrogenase/fumarate reductase $\mathrm{e}^{39}$. The similarity bitscores of these subunits from Pde toward eukaryotes (Figure $5 \mathrm{~b}$ panels 11-12) showed the expected absence of these subunits from the AMIs in contrast to their presence in a variety of MTEs. Comparison of panels 9-12 indicated that Ein and Enh exchanged their mitochondrial FrdB and SdhA for mitosomal $[\mathrm{Fe}]$ hydrogenase and PFO in their adaptation to anaerobiosis. The source of their [Fe] hydrogenase and PFO, be it AGA from Clostridium, or some ancestral AMI or Amoebozoa remains an open question.

When 151 different alphaproteobacteria together with some other bacteria were compared with respect to their similarity bitscores toward the mtDNA-encoded proteins of different eukaryotes including the bacteria-like Ram $^{40}$ (Figure 5c top panel; Supplementary Figure S2), the bitscores of Tis ranked the highest, followed by another Rhodospirillales Azo, suggesting that the endosymbiont precursor of mitochondria was either Tis or a close relative, in accord with the placement of the Tis branch next to the mitochondrial branch on the alphaproteobacterial tree ${ }^{41}$. However, a range of other alphaproteobacteria either contributed mtDNA-encoded proteins or showed overlapping similarities with Tis proteins; among the nonalphaproteobacteria, only Rso and Xca exhibited some modest bitscores. These findings were indicative of a shared genetic propensity of the alphaproteobacterial group for symbiosis with the eukaryotes, as in the case of the graded propensities of different strains of Nostoc for ectosymbiosis with Anthoceros ${ }^{42}$. Such shared propensity promoted symbioses between the eukaryotic lineage and multiple alphaproteobacterial species, thereby allowing entry of their genes into some eukaryotes to replace or recombine with the Tis-genes in mtDNA. Among human mtDNA-encoded proteins HM1-11, Ehrlichia and Rpr yielded the highest bitscores 
toward HM2 and HM3 respectively (middle panel), suggesting that their proteins had replaced original Tis sequences. Since Ehrlichia and Rpr were both infectious for humans, the replacements might have occurred during infection. Replacements/recombinations also could be a factor in the exceptionally diverse sources of Blastocystis mtDNA-encoded proteins and host-related SSU rRNA subtypes ${ }^{43}$.

\section{Discussion}

Because the $1.5 \mathrm{Mb}$ archaeal parent genome of Abo was much smaller than for example the 11.2 Mb eukaryotic genome of Gla, recruitment of exogenous prokaryotic genes into the Abo genome was a top priority right from the start of eukaryogenesis. Too small to house an upfront endosymbiont, the archaal host had to depend on AGA and ectosymbiosis for acquisition of archaeal proteins from such prominent sources as Mco, Pfu, Mac, TACKs and Asgards into the AMIs (Figure 4). The modest numbers of bacterial proteins from Tis, Rru, Bja, Atu, Cvo, Cur, Rso, Aba, Xca, Pel, Dra,Tht, Bsu, Syn, Ssp, Tte, Hth, Cpo, Mau and Pmo detected in various AMIs pointed to gene acquisitions by the AMIs from these bacteria through ectosymbioses. The Eco proteins was a special case: they were present in modest numbers in the microsporidial Slo-Oco, but increased sharply from Ebi onward to Hsa. Thus Eco was likely an ectosymbiont with modest contribution of proteins at first, but became later either an endosymbiont, or an especially effective or sustained ectosymbiont with an enhanced influx. In any event, the presence of Eco proteins in both the AMIs and MTEs was consistent with the Eco-like str-S10-spc-alpha operons, as well as clustering and organization of $r p s$ and $r p l$ genes in some mtDNAs ${ }^{44}$.

The wide ranging influxes of prokaryotic genes through AGA and ectosymbiosis enabled the AMIs to expand in genome size, and in cell volume akin to the development of giant cell size by the archaeon Giganthauma karukerense through ectosymbiosis with a gammaproteobacterium $^{45}$. The convergence of enlarged cell volume, an expanded genome with ESPs from Asgards, TACKs and other prokaryotes, and an exceptionally flexible cell membrane that could enwrap symbionts with its blebs ${ }^{31}$ equipped the AMIs for the development of phagocytosis ${ }^{46,47}$. This resulted in endosymbioses with Tis marked by a flood of Tis proteins that greatly exceeded the protein influxes from various ectosymbionts; and still later with a cyanobacterium marked by a comparable flood of Syn-like proteins into the algae Tps, Esi, Bpr, Gth and Cme. These endosymbioses gave the MTEs the twin biofuel engines of oxidative phosphorylation and photosynthesis that drove their relentless expansion for the next two giga years. In addition, the Rru, Bja, Atu, Cvo, Pde, Cur, Rso, Aba, and Xca proteins all grew to pronounced levels in the MTEs that signaled their endosymbioses or highly effective/sustained ectosymbioses with the eukaryotes. Notably, the mtDNA-encoded proteins of numerous eukaryotes notched higher bitscores toward Tis than Pde, Bja, Bsu, Filomicrobium and Halocynthiibacter (Figure 5c top panel), whereas the nuclear encoded PycA, NirB, AceF, AHP1 and FMR2 of some eukaryotes notched higher bitscores toward Pde, Bja, Bsu, Filomicrobium or Halocynthiibacter than Tis (bottom panel). Therefore the eukaryotes plausibly formed separate symbioses with Tis, Pde, Bja, Bsu and others; while the Tisendosymbiont served as precursor to mitochondria, the symbioses with Pde, Bja, Bsu and others introduced a variety of useful proteins into the eukaryotes.

With respect to the ectosymbiosis-based mechanism (Figure 3), the role of Abo as archaeal parent and the presence of numerous ectosymbionts detected in Figure 4 confirmed the postulated interactions of archaeal parent with ectosymbionts in various stages starting from 
222 Stage 1. The contributions of [Fe] hydrogenase and PFO by Tte confirmed the postulate of archaeal-ectosymbiont collaborations to build mitosome and hydrogenosome in Stage 2 and Stage 3 respectively. Identification of a Tis-endosymbiont as precursor of mitochondria confirmed the postulate of archaeal-alphaproteobacterial endosymbiosis in Stage 4. Clearly, a horde of micron-size prokaryotes generated the Eukarya domain with their genes through accelerated gene adoption, ectosymbiosis and endosymbiosis, and all four stages of the ectosymbiotic mechanism were verified by fluxes of prokaryotic protein-coding genes into the eukaryotes $^{48}$.

The unique crown-capped structure of the SSU rRNA tree ${ }^{4}$ stands as a monument to the cataclysmic effects of oxygen on eukaryotic evolution. That Abo and the lowest branching AMIs were anaerobic suggests that eukaryogenesis started in the anoxic Archean Eon. Oxygenproducing cyanobacteria evolved at or before 2.7 Gya, providing an atmosphere containing a trace amount of free oxygen that persisted up to $2.4 \mathrm{Gya}$. It made possible sterol biosynthesis in eukaryotes, leaving sterane footprints of eukaryotes in 2.7-Gya rocks even though it fell far short of supporting aerobic metabolism ${ }^{49}$. The increased levels of oxygen during the Great Oxidation Event at about 2.4 Gya in early Proterozoic Eon was a grave hazard to the anaerobic bacteria, archaea and eukaryotes, for the incongruence between enzyme distribution in aerobic metabolic pathways and organismal speciation also indicated that all three domains of organisms were already formed at that juncture. In response to the rising oxygen, all the surviving species from that era built oxic metabolic networks in addition to their earlier anoxic ones $^{50}$. Among the species on the eukaryotic SSU rRNA tree, which was by necessity an allAMI tree stemming from an amitochondriate LECA, the lowest branching species with their limited resources probably built oxic networks that were little more than $3-4 \%$ their anoxic networks as exemplified by the anaerobic prokaryotes Tma, Mka and Tte. As a result, these lowest branchers remained as AMIs, were saddled with reduced competitiveness, and had to choose between parasitism and confinement to anaerobic niches. Their long branches on the present-day tree could be indicative of their arduous evolutionary journeys to stay alive. Higher up on the tree, Pfa built an oxic network equal to about $5 \%$ of its anoxic one and managed to possess mitochondria, but still ended up as a parasite. In contrast, the elaborate species at the top of the tree acquired mitochondria and built large oxic networks greater than $20 \%$ of the anoxic network in Sce, $30 \%$ in Cel, $40 \%$ in Arabidopsis thaliana, or 50-60\% in the mammals. Endowed with large oxic networks and plentiful ATP from oxidative phosphorylation, they launched into adaptive radiation to form the spikes on the crown of the tree. The accomplishments of the Hsa lineage began when it became one of the spikes.

\section{Methods}

\section{Source of Data and Materials}

258 Protein sequences were retrieved from NCBI GenBank release 231 259 (ftp://ftp.ncbi.nlm.nih.gov/genomes/) ${ }^{51}$. Assemblies of species analyzed were given in 260 Supplementary Table S1. Quality checked SSU rRNA sequences were downloaded from the 261 SILVA database release 132 (https://www.arb-silva.de/) ${ }^{52}$. The accession numbers of SSU 262 rRNAs analyzed were available in Supplementary File S1. Eukaryotic mitochondrial DNA263 encoded protein sequences were retrieved from the RefSeq mitochondrial reference genomes in the NCBI Protein database (https://www.ncbi.nlm.nih.gov/protein) ${ }^{53}$. 
The SSU rRNAs of 47 eukaryotes and 3 archaeons (Abo, Mja, Lok) as outgroup were aligned in Clustal Omega ${ }^{54}$, and the tree was constructed using RAxML with an GTR+GAMMA+I model, with 1000 bootstraps ${ }^{55}$. The final maximum-likelihood tree with bootstrap support values is available in Supplementary File S2.

\section{Hsp70-type Sequences}

The proteome of each species analyzed was searched against the database of Clusters of Orthologous Groups of proteins $(\mathrm{COG})^{34}$ using RPSBLAST ${ }^{56}$ at a threshold set by the '-evalue' flag at 0.01. Potential Hsp70 sequences in each species were identified as those mapped to COG0443 (Molecular chaperone DnaK or Hsp70). Among the potential Hsp70 sequences, one sequence was manually selected, if available, for each of the Group I_KP motif and the Group II NGDAWV motif. See Supplementary Table S2 for sequence accession numbers.

\section{Estimation of Nuclear or Mitochondrial Proteome Similarity Bitscores}

When comparing proteome similarities for Figures 4 and $5 \mathrm{c}$ top panel, the proteomes of all subject species were used to construct a local BLAST database using makeblastdb ${ }^{56}$, and every query proteome was searched against the local database using BLASTP with a BLOSUM62 matrix and thresholds setting to e-value $<1 \times 10^{-5}$, percent identity $>25 \%$ and query coverage $>$ $50 \%$. Only the query and subject sequences that were the best-match of each other, viz. when query sequence $n$ from species 1 exhibited the highest bitscore toward subject sequence $m$ among all proteins of species 2 and vice versa, were included in the estimation of interproteome similarity, which was given by the sum of BLASTP bitscores of all such bestmatched proteins between the two proteomes. The data for nuclear and mitochondrial proteome similarities is given in Supplementary Tables S3 and S4a respectively. The bacterial/archaeal protein ratio (R) was estimated as $R=\sum_{1}^{i} N_{b} / \sum_{1}^{j} N_{a}$, where $i$ was the number of proteins in a eukaryotic proteome that could be blasted to the proteins of any of the 33 bacterial species analyzed in Figure 4; and $\boldsymbol{N}_{\boldsymbol{b}}$ was the highest bitscore so obtained. Likewise, $\boldsymbol{j}$ was the number of proteins in the same eukaryotic proteome that could be blasted to any of the also 33 archaeal species; and $\boldsymbol{N}_{\boldsymbol{a}}$ was the highest bitscore so obtained.

\section{Similarity of COGs between eukaryotes and prokaryotes}

Best-matched query and subject sequences passing BLASTP thresholds of e-value $<1 \times 10^{-5}$, percent identity $>25 \%$ and query coverage $>50 \%$ that were mapped to the same COG using RPSBLAST at a threshold of e-value $<0.01$ furnished data for Figures $5 \mathrm{a}, 5 \mathrm{~b}$ and $5 \mathrm{c}$ middle and bottom panels. For Figure 5b, the bitscores of 10 out of 196 protein pairs that did not pass the percent-identity or query-coverage thresholds but showed BLASTP e-values $<1 \times 10^{-10}$ were also included (see Supplementary Table S5 for the e-values, percent identities and query coverages for Figure 5b).

\section{Data availability}

302 All the data generated in this study were included in the supplementary information files. 


\section{References}

304 1. Martin, W. F., Garg, S. \& Zimorski, V. Endosymbiotic theories for eukaryote origin. Philos. Trans. R. Soc. Lond., B, Biol. Sci. 370, 20140330, doi:10.1098/rstb.2014.0330 (2015).

2. Vossbrinck, C., Maddox, J., Friedman, S., Debrunner-Vossbrinck B. A. \& Woese C. R. Ribosomal RNA sequence suggests microsporidia are extremely ancient eukaryotes. Nature 326, 411-414 (1987).

3. Cavalier-Smith, T. The origin of eukaryotic and archaebacterial cells. Ann. N. Y. Acad. Sci. 503, 1754 (1987).

4. Sogin, M. L. \& Silberman, J. D. Evolution of the protists and protistan parasites from the perspective of molecular systematics. Int. J. Parasitol. 28, 11-20 (1998).

5. Roger, A. J. Reconstructing early events in eukaryotic evolution. Am. Nat. 154, S146-S163 (1999).

6. Van der Giezen, M. Hydrogenosomes and mitosomes: conservation and evolution of functions. $J$.

7. Dacks, J. B. et al. The changing view of eukaryogenesis-fossils, cells, lineages and how they all come

8. Long, X., Xue, H. \& Wong, J. T. Descent of Bacteria and Eukarya from an archaeal root of life. Evol.

9. Rogozin, I. B., Basu, M. K., Csuros, M. \& Koonin, E. V. Analysis of rare genomic changes does not support the unikont-bikont phylogeny and suggests cyanobacterial symbiosis as the point of primary radiation of eukaryotes. Genome Biol Evol 1, 99-113 (2009).

10. Cavalier-Smith, T. Kingdoms Protozoa and Chromista and the eozoan root of the eukaryotic tree. Biol. Lett. 6, 342-345 (2010).

11. Katz, L. A., Grant, J. R., Parfrey, L. W. \& Burleigh, J. G. Turning the crown upside down: gene tree parsimony roots the eukaryotic tree of life. Syst. Biol. 61, 653-660 (2012).

12. He, D. et al. An alternative root for the eukaryote tree of life. Curr. Biol. 24, 465-470 (2014).

13. McInerney, J., Pisani, D. \& O'Connell, M. J. The ring of life hypothesis for eukaryote origins is supported by multiple kinds of data. Philos. Trans. R. Soc. Lond., B, Biol. Sci. 370, 20140323, doi:10.1098/rstb.2014.0323 (2015).

14. Morin, L. Long branch attraction effects and the status of "basal eukaryotes": phylogeny and structural analyses of the ribosomal RNA gene cluster of the free-living diplomonad Trepomonas agiles. J. Eukaryot. Microbiol. 87, 167-77 (2000).

15. Bergsten, J. A review of long-branch attraction. Cladistics 21, 163-193 (2005).

16. Cavalier-Smith, T. Multigene phylogeny resolves deep branching of Amoebozoa. Mol. Phylogenet. Evol. 83, 293-304 (2015). 
19. Van de Peer, Y., Rensing, S. A., Maier, U. G. \& De Wachter, R. Substitution rate calibration of small subunit ribosomal RNA identifies Chlorarachniophyte endosymbionts as remnants of green algae. Proc. Natl. Acad. Sci. U.S.A. 93, 7732-36 (1996).

20. Germot, A., Philippe, H. \& Le Guyader, H. Evidence for loss of mitochondria in Microsporidia from a mitochondrial-type HSP70 in Nosema locustae. Mol. Biochem. Parasitol. 87, 159-168 (1997).

21. Troemel, E. R. \& Becnel, J. J. Genome analysis and polar tube firing dynamics of mosquito-infecting microsporidia. Fungal Genet. Biol. 83, 41-44 (2015).

22. Capella-Gutierrez, S., Marcet-Houben, M. \& Gabaldon, T. Phylogenomics supports Microsporidia as the earliest diverging clade of sequenced fungi. BMC Biol. 10, 47 (2012).

23. Arisue, N., Sanchez, L. B., Weiss, L. M., Muller, M. \& Hashimoto, T. Mitochondrial-type hsp70 genes of the amitochindriate protists Giardia intestinalis, Entamoeba histolytica and two microsoporidia. Parasitol. Int. 51, 9-16 (2002).

24. Esser, C. et al. A genome phylogeny for mitochondria among alpha-proteobacteria and a predominantly eubacterial ancestry of yeast nuclear genes. Mol. Biol. Evol. 21, 1643-60 (2004).

25. Rochette, N. C., Brochier-Armanet, C. \& Gouy, M. Phylogenomic test of the hypotheses for the origin of eukaryotes. Mol. Biol. Evol. 31, 832-845 (2014).

26. Koonin, E. V. \& Yutin, N. The dispersed archaeal eukaryome and the complex archaeal ancestor of eukaryotes. Cold Spring Harb Perspect Biol 6, a016188 (2014).

27. Akanni W. A. et al. Horizontal gene flow from eubacteria to archaebacteria and what it means for our understanding of eukaryogenesis. Philos. Trans. R. Soc. Lond., B, Biol. Sci. 370, 20140337, doi: 10.1098/rstb.2014.0337(2015).

28. Clark, C. G. \& Roger, A. J. Direct evidence for secondary loss of mitochondria in Entamoeba histolytica. Proc. Natl. Acad. Sci. U.S.A. 92, 6518-21 (1995).

29. Pyrih, J. et al. Minimal cytosolic iron-sulfur cluster assembly machinery of Giardia intestinalis is partially associated with mitosome. Mol. Microbiol. 102, 701-714 (2016).

30. Devos, D. et al. Components of coated vesicles and nuclear pore complex share a common molecular architecture. PLoS Biol. 2, e380, doi: 10.1371/journal.pbio.0020380 (2004).

31. Reysenbach, A. L. et al. A ubiquitous thermoacidophilic archaeon from deep-sea hydrothermal vents. Nature 442, 444-447 (2006).

32. Danovaro, R. et al. The first metazoa living in permanently anoxic conditions. BMC Biol. 8, 30 (2010).

33. Kurland, C. G., Canback, B. \& Berg, O. G. Horizontal gene transfer: a critical view. Proc. Natl. Acad. Sci. U.S.A. 100, 9658-62 (2003).

34. Tatusov, R. L., Galperin, M. Y., Natale, D. A. \& Koonin, E. V. The COG database: a tool for genomescale analysis of protein functions and evolution. Nucleic Acids Res. 28, 33-36 (2000).

35. Eme, L., Spang, A., Lombard, J., Stairs, C. W. \& Ettema, T. J. G. Archaea and the origin of eukaryotes. Nat. Rev. Microbiol. 15, 711-723 (2017).

36. Hartman, H. \& Fedorov, A. The origin of the eukaryotic cell: a genomic investigation. Proc. Natl. Acad. Sci. U.S.A. 99, 1420-25 (2002). 
37. Gentekaki, E. et al. Extreme genome diversity in the hyper-prevalent parasitic eukaryote Blastocystis. PLoS Biol. 15, e2003769, doi:10.1371/journal.pbio.2003769 (2017).

38. Whatley, J. M., John, P. \& Whatley, F. R. From extracellular to intracellular: the establishment of mitochondria and chloroplasts. Proc. R. Soc. Lond., B, Biol. Sci. 204, 165-187 (1979).

39. Tielens, A. G. M., Rotte, C., van Hellemond, J. J. \& Martin, M. Mitochondria as we don't know them. Trends Biochem. Sci. 27, 565-572 (2012).

40. Burger, G., Gray, M. W., Forget, L. \& Lang, B. F. Strikingly bacteria-like and gene-rich mitochondrial genomes throughout jakobid protists. Genome Biol Evol 5, 418-438 (2013).

41. Martijn, J., Vosseberg, J., Guy, L., Offre, P. \& Ettema, T. J. G. Deep mitochondrial origin outside the sampled alphaproteobacteria. Nature 557, 101-105 (2018).

42. Enderlin, C. S. \& Meeks, J. C. Pure culture and reconstitution of the Anthoceros-Nostoc symbiotic association. Planta. 158, 157-165 (1983).

43. Stensvold, C. R. et al. Subtype distribution of Blastocystis isolates from synanthropic and zoo animals and identification of a new subtype. Int. J. Parasitol. 39, 473-479 (2009).

44. Gray, M. W. Mitochondrial evolution. Cold Spring Harb Perspect Biol 4, a011403, doi:10.1101/cshperspect.a011403 (2012).

45. Muller, F., Brissac, T., Le Bris, N., Felbeck, H. \& Gros, O. First description of giant Archaea (Thaumarchaeota) associated with putative bacterial ectosymbionts in a sulfidic marine habitat. Environ. Microbiol. 12, 2371-83 (2010).

46. Yutin, N., Wolf, M. Y., Wolf, Y. I. \& Koonin, E. V. The origins of phagocytosis and eukaryogenesis. Biol. Direct 4, 9, doi:10.1186/1745-6150-4-9 (2009).

47. Martin, W. F., Tielens, A. G. M., Mentel, M., Garg, S. G. \& Gould, S. B. The physiology of phagocytosis in the context of mitochondrial origin. Microbiol. Mol. Biol. Rev. 81, e0008-17 (2017).

48. Wong, J. T., Xue, H. \& Long, X. An ectosymbiosis-based mechanism of eukaryogenesis. Preprint (2020).

49. Kasting, J. F. The primitive Earth. In Prebiotic Evolution and Astrobiology eds. Wong, J. T. \& Lazcano, A. 57-63 Landes Bioscience (2009).

50. Raymond, J. \& Segre, D. The effect of oxygen on biochemical networks and the evolution of complex life. Science 311, 1764-67 (2006).

51. Clark, K., Karsch-Mizrachi, I., Lipman, D. J., Ostell, J. \& Sayers, E. W. GenBank. Nucleic Acids Res. 44, D67-72, doi:10.1093/nar/gkv1276 (2016).

52. Quast, C. et al. The SILVA ribosomal RNA gene database project: improved data processing and webbased tools. Nucleic Acids Res. 41, D590-596, doi:10.1093/nar/gks1219 (2013).

53. O'Leary, N. A. et al. Reference sequence (RefSeq) database at NCBI: current status, taxonomic expansion, and functional annotation. Nucleic Acids Res. 44, D733-745, doi:10.1093/nar/gkv1189 (2016).

54. Sievers, F. \& Higgins, D. G. Clustal Omega for making accurate alignments of many protein sequences. Protein Sci. 27, 135-145, doi:10.1002/pro.3290 (2018). 
55. Stamatakis, A. RAxML version 8: a tool for phylogenetic analysis and post-analysis of large phylogenies. Bioinformatics 30, 1312-1313, doi:10.1093/bioinformatics/btu033 (2014).

417 56. Camacho, C. et al. BLAST+: architecture and applications. BMC Bioinform. 10, 421,

\section{Acknowledgements}

420 The authors would like to acknowledge the University Grant Council for funding support to 421 Applied Genomics Center [No. 5112-703-0110D-42002].

\section{Author contributions}

423 J.T.W. and H.X. conceived the study; X.L. collected the data and performed computational 424 analysis; and J.T.W., X.L. and H.X wrote the paper. All authors read and approved the final 425 manuscript.

\section{Competing interests}

427 The authors declare that there is no conflict of interest with respect to the research, authorship, 428 and/or publication of this article.

\section{Materials \& Correspondence}

430 Correspondence to J. Tze-Fei Wong. 
Table 1. Partial list of species analysed.

\begin{tabular}{|c|c|c|c|}
\hline \multicolumn{2}{|c|}{ ABBR. SPECIES NAME } & \multicolumn{2}{|c|}{ ABBR. SPECIES NAME } \\
\hline \multicolumn{2}{|c|}{ ARCHAEA } & \multicolumn{2}{|c|}{ BACTERIA (CONTINUED) } \\
\hline Abo & Aciduliprofundum boonei & Spn & Streptococcus pneumoniae \\
\hline Acf & Aciduliprofundum sp. MAR08-339 & Ssp & Sporanaerobacter sp. NJN-17 \\
\hline Afu & Archaeoglobus fulgidus & Syn & Synechocystis sp. PCC 6803 \\
\hline Aia & Acidilobus sp. $7 A$ & Tht & Thermobaculum terrenum \\
\hline Alt & C.Altiarchaeales archaeon & Tis & Tistrella mobilis \\
\hline Ape & Aeropyrum pernix & Tma & Thermotoga maritima \\
\hline Bat & C.Bathyarchaeota archaeon & Tte & Thermoanaerobacter tengcongensis \\
\hline $\mathrm{Csu}$ & C.Caldiarchaeum subterraneum & Xca & Xanthomonas campestris \\
\hline Csy & Cenarchaeum symbiosum & \multicolumn{2}{|c|}{ EUKARYA } \\
\hline $\mathrm{Fac}$ & Ferroplasma acidiphilum & $\overline{\mathrm{Aal}}$ & $\overline{\text { Anncaliia algerae }}$ \\
\hline Ffo & Fervidicoccus fontis & Acc & Acanthamoeba castellanii \\
\hline Hal & Halobacterium salinarum & Ago & Andalucia godoyi \\
\hline Hei & C.Heimdallarchaeota archaeon & Asu & Acytostelium subglobosum \\
\hline Hgi & Haloferax gibbonsii & Bbo & Babesia bovis \\
\hline Hla & Halobiforma lacisalsi & Bde & Batrachochytrium dendrobatidis \\
\hline Kcr & C.Korarchaeum cryptofilum & Bho & Blastocystis hominis \\
\hline Lok & Lokiarchaeum sp. GC14 75 & Bpr & Bathycoccus prasinos \\
\hline Mac & Methanosarcina acetivorans & Bs1 & Blastocystis sp. subtype 1 \\
\hline Mbo & Methanoregula boonei & Bs3 & Blastocystis sp. subtype 3 \\
\hline Mco & Methanocella conradii & Bs4 & Blastocystis sp. subtype 4 \\
\hline Mes & C.Methanosuratus sp. & Cel & Caenorhabditis elegans \\
\hline Min & C.Methanomassiliicoccus intestinalis & Cme & Cyanidioschyzon merolae \\
\hline Mja & Methanocaldococcus jannaschii & Cne & Cryptococcus neoformans \\
\hline Mka & Methanopyrus kandleri & Cpa & Cryptosporidium parvum \\
\hline Mnt & Methanonatronarchaeum thermophilum & Ddi & Dictyostelium discoideum \\
\hline Mph & Methanophagales archaeon & Dme & Drosophila melanogaster \\
\hline Mte & C.Methanoplasma termitum & Dpu & Dictyostelium purpureum \\
\hline Nca & C.Nitrosocaldus cavascurensis & Dre & Danio rerio \\
\hline Nga & C.Nitrososphaera gargensis & $\mathrm{Eae}$ & Edhazardia aedis \\
\hline Odi & C.Odinarchaeota archaeon & Ebi & Enterocytozoon bieneusi \\
\hline $\mathrm{Pae}$ & Pyrobaculum aerophilum & Ecu & Encephalitozoon cuniculi \\
\hline Pfu & Pyrococcus furiosus & Ein & Entamoeba invadens \\
\hline Psy & C.Prometheoarchaeum syntrophicum & Enh & Entamoeba histolytica \\
\hline Sso & Saccharolobus solfataricus & Esi & Ectocarpus siliculosus \\
\hline $\mathrm{Tac}$ & Thermoplasma acidophilum & Gin & Giardia intestinalis \\
\hline Tho & C.Thorarchaeota archaeon & Gla & Giardia lamblia \\
\hline Tvo & Thermoplasma volcanium & Gth & Guillardia theta \\
\hline Woa & C.Woesearchaeota archaeon & Has & Homo sapiens \\
\hline \multicolumn{2}{|c|}{ BACTERIA } & $\operatorname{Imu}$ & Ichthyophthirius multifiliis \\
\hline$\overline{\mathrm{Aae}}$ & $\overline{\text { Aquifex aeolicus }}$ & Lbi & Laccaria bicolor \\
\hline Aba & Acinetobacter baumannii & Mbr & Monosiga brevicollis \\
\hline Atu & Agrobacterium tumefaciens & Mci & Mucor circinelloides \\
\hline Azo & Azospirillum sp. M2T2B2 & Mon & Monocercomonoides sp. PA203 \\
\hline Bap & Buchnera aphidicola & Mpa & Marchantia paleacea \\
\hline Bja & Bradyrhizobium japonicum & Nbo & Nosema bombycis \\
\hline Blo & Bifidobacterium longum & Ner & Neurospora crassa \\
\hline Bsu & Bacillus subtilis & Ngr & Naegleria gruberi \\
\hline Cex & Caldisericum exile & Oco & Ordospora colligate \\
\hline Cje & Campylobacter jejuni & Per & Perkinsela sp. CCAP 1560/4 \\
\hline Cpo & Cloacibacillus porcorum & Pfa & Plasmodium falciparum \\
\hline Cur & Curvibacter sp. & Pte & Paramecium tetraurelia \\
\hline Cvo & Chelativorans sp. BNC1 & Ram & Reclinomonas Americana \\
\hline Dra & Deinococcus radiodurans & Sap & Saprolegnia parasitica \\
\hline Dth & Dictyoglomus thermophilum & Sce & Saccharomyces cerevisiae \\
\hline Eco & Escherichia coli & Slo & Spraguea lophii \\
\hline Hth & Hungateiclostridium thermocellum & Spo & Schizosaccharomyces pombe \\
\hline $\mathrm{Kae}$ & Klebsiella aerogenes & Spu & Spizellomyces punctatus \\
\hline Kol & Kosmotoga olearia & Sra & Strongyloides ratti \\
\hline Mau & Mahella australiensis & Ssa & Spironucleus salmonicida \\
\hline Mca & Macrococcus caseolyticus & Tbr & Trypanosoma brucei \\
\hline Mtu & Mycobacterium tuberculosis & Tgo & Toxoplasma gondii \\
\hline Pde & Paracoccus denitrificans & Tps & Thalassiosira pseudonana \\
\hline Pel & Pelobacter sp. SFB93 & Trh & Trachipleistophora hominis \\
\hline Pmo & Petrotoga mobilis & Trv & Trichomonas vaginalis \\
\hline Rpr & Rickettsia prowazekii & Tth & Tetrahymena thermophila \\
\hline Rru & Rhodospirillum rubrum & $\mathrm{Ttr}$ & Thecamonas trahens \\
\hline Rso & Ralstonia solanacearum & $\mathrm{Vcu}$ & Vavraia culicis \\
\hline
\end{tabular}

432 Note: C. in front of species name stands for Candidatus. Detailed species information is given in 433 Supplementary Table S1. 


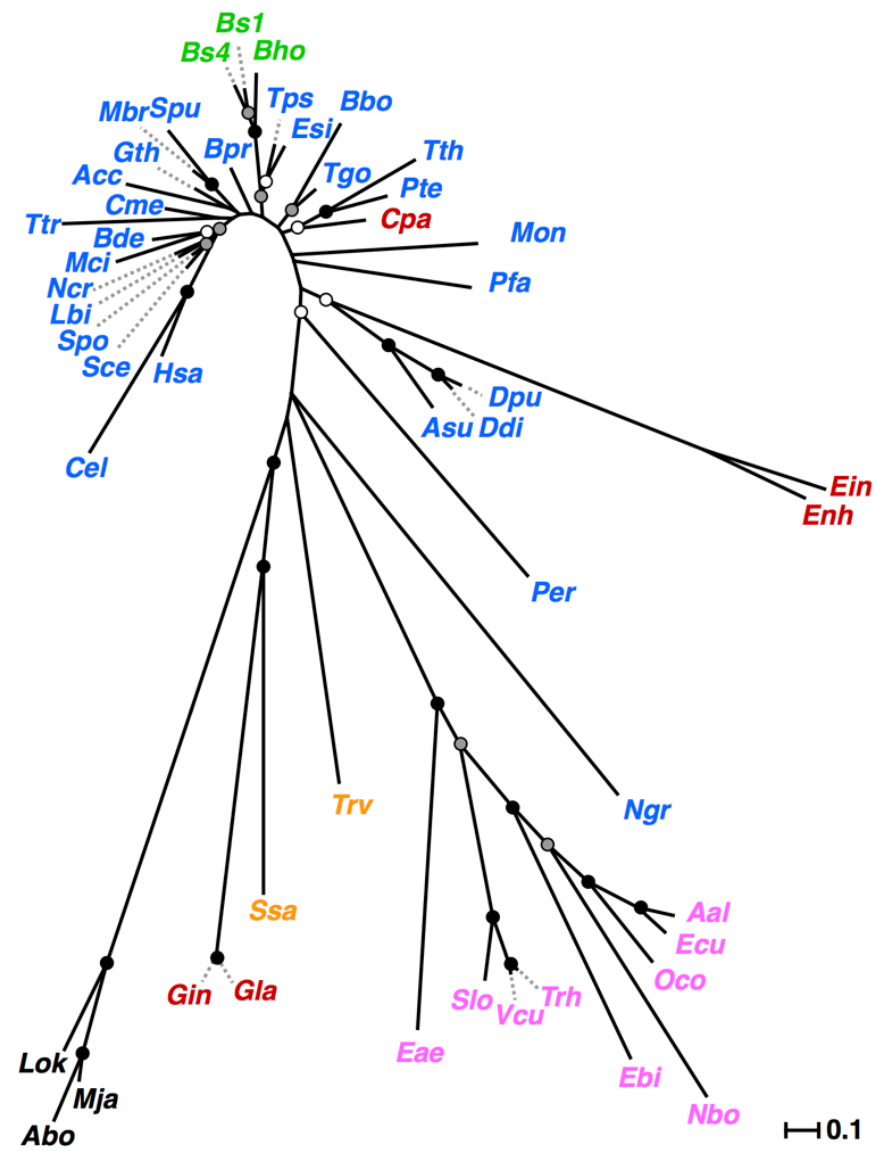

436 Figure 1. Maximum-likelihood SSU rRNA tree. Eukaryotes with mitochondria, and also Mon, are labeled in blue; Microsporidia in pink; non-Microsporidia eukaryotes with mitosome in red; eukaryotes with hydrogenosome in orange; eukaryotes with MRO and part of mitochondria in green; and an archaeal outgroup in black. Nodes with $>90 \%$ bootstrap support are represented by black circles, $>70 \%$ grey circles, $>50 \%$ open circles, and $\leq 50 \%$ without circle. 


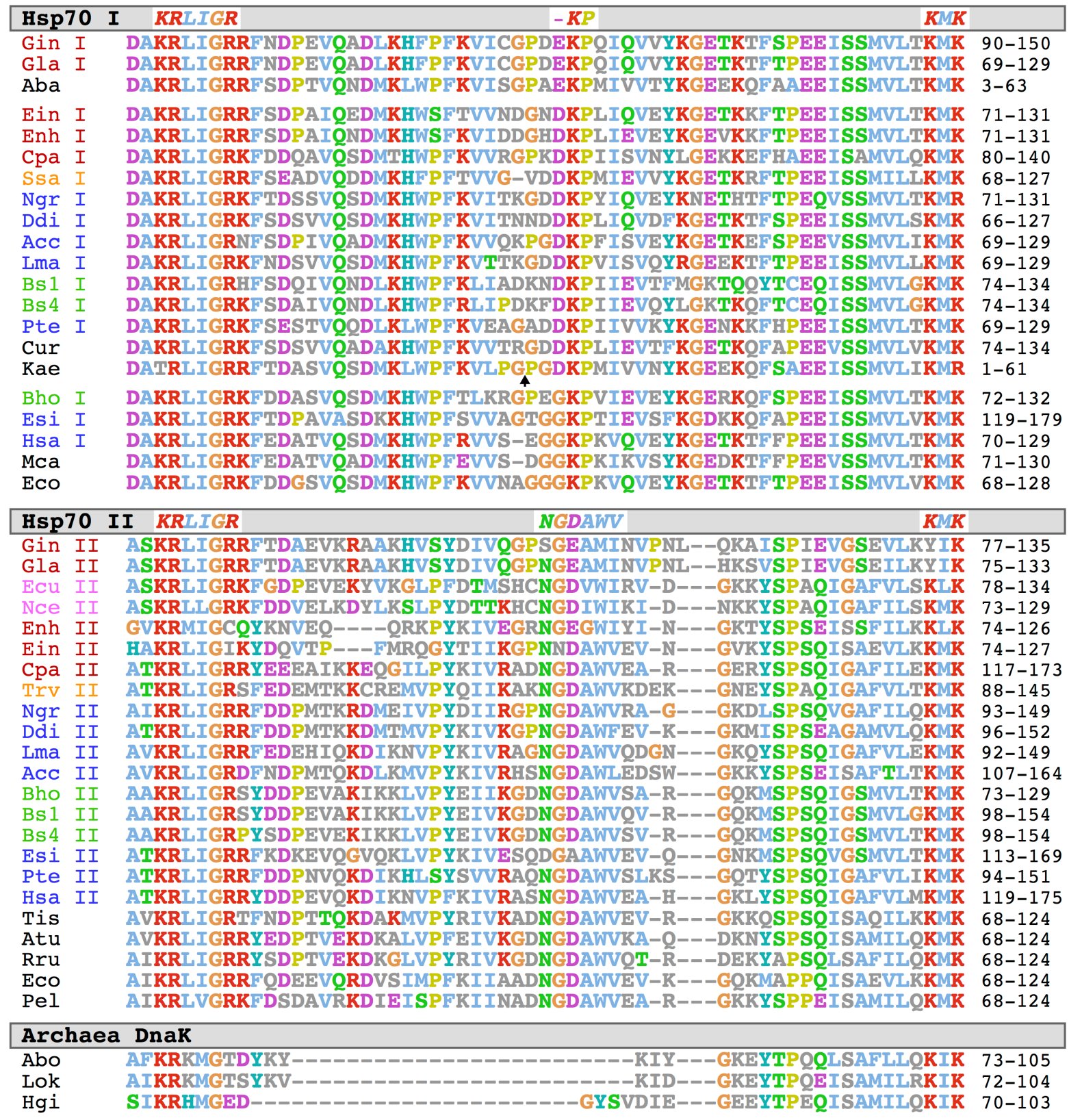

444 Figure 2. Hsp70 sequence motifs. Group-I sequences with EKP/DKP/GKP motifs and Group445 II sequences with NGDAWV motif in eukaryotic Hsp70 sequences and prokaryotic DnaK 446 sequences were aligned using the KRLIGR and KMK motifs at the 5' and 3' ends of segment 447 as positional markers. The numbers on the right indicate the positions of amino acid residues 448 of each segment within its Hsp70 or DnaK sequence. Names of eukaryotes are colored as in 449 Figure 1, and names of prokaryotes are colored black. The arrow indicates the position of an 450 additional amino acid residue on the Ddi I sequence. 


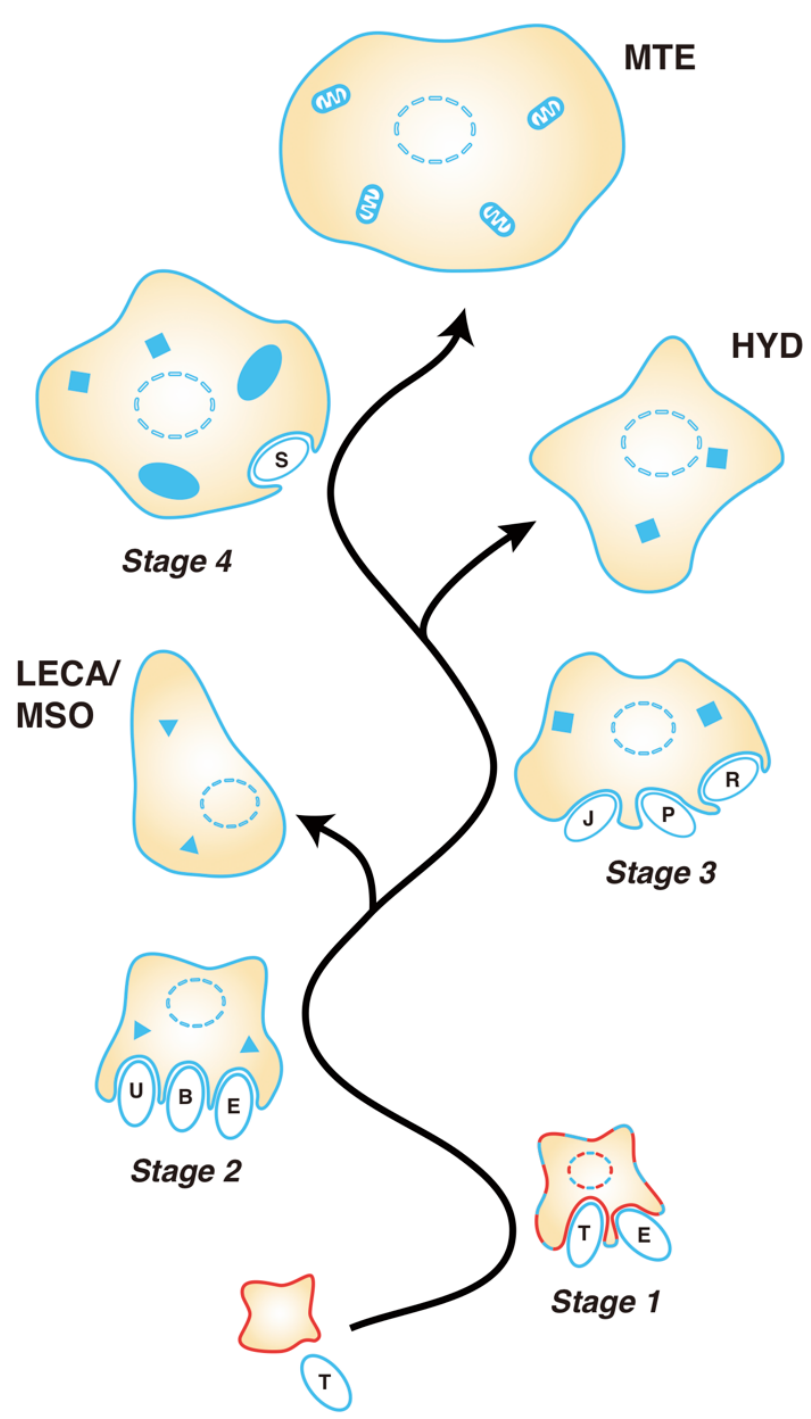

453 Figure 3. Ectosymbiotic mechanism of eurkaryogenesis. Stages 1-4 are developmental stages. Ellipse enclosed by dashed line represents cell nucleus. The membranes of archaeal parent are colored red and bacterial membranes colored blue. Solid triangles are mitosomes, and solid squares hydrogenosomes. MSO, HYD and MTE represent mitosome-containing, hydrogenosome-containing and mitochondria-containing eukaryotes. The letters T, E, U, B, J, $\mathrm{P}, \mathrm{R}$ and $\mathrm{S}$ stand for Tte, Eco, Rru, Bsu, Bja, Pde, Rso and Tis respectively, representing a small fraction of the bacteria taking part as ectosymbionts in the mechanism. Solid ellipses denote endosymbionts, and ellipses with cristae mitochondria. 


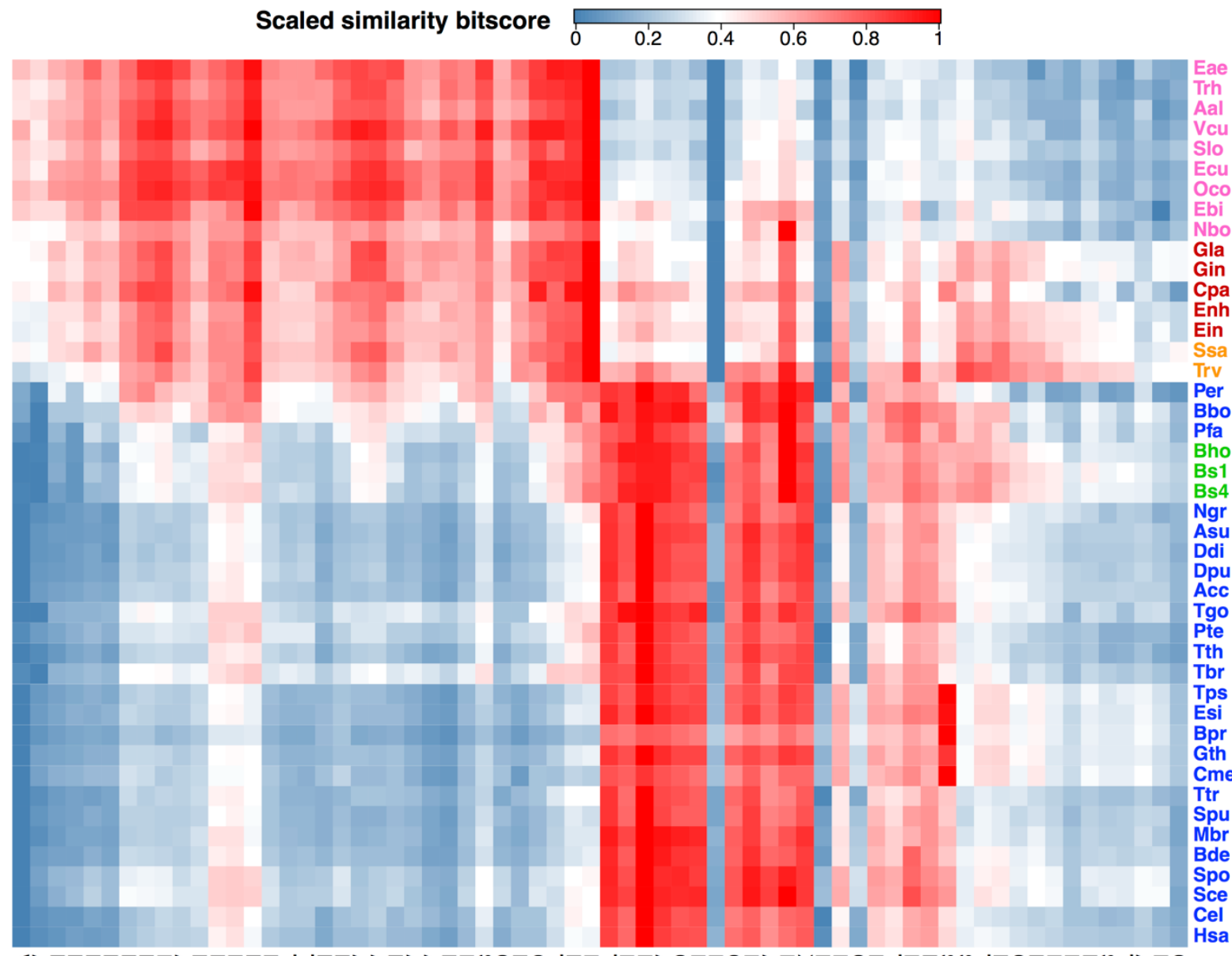

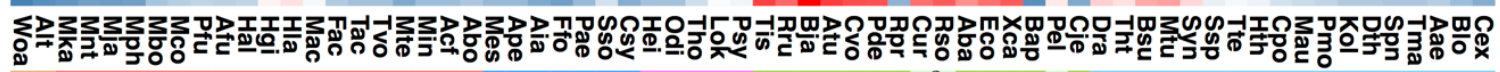

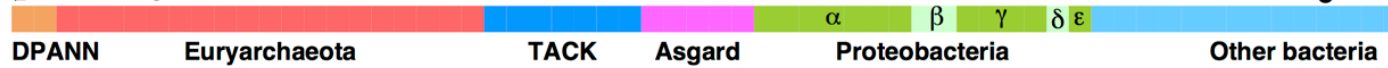

Figure 4. Heat map of inter-proteome similarity bitscores between eukaryotes (y-axis) and prokaryotes (x-axis). For each eukaryotic proteome, its similarity bitscores toward different prokaryotes were scaled linearly from 0 to 1.0 on the thermal scale, with zero (blue) marking the minimum, and 1.0 (red) the maximum bitscores. The names of different eukaryotes are colored as in Figure 1; and the colour bands on the x-axis indicate different groupings of prokaryotic species. The raw bitscores for the heat map are shown in Supplementary Table S3. 
a
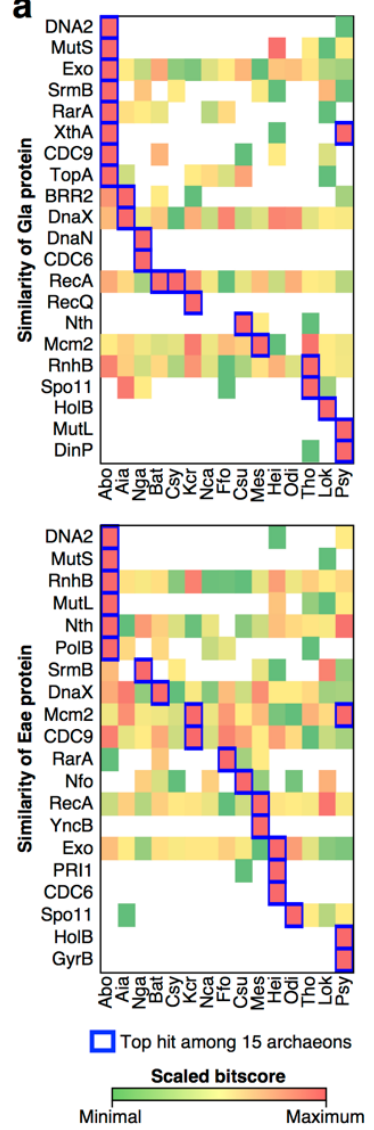

b

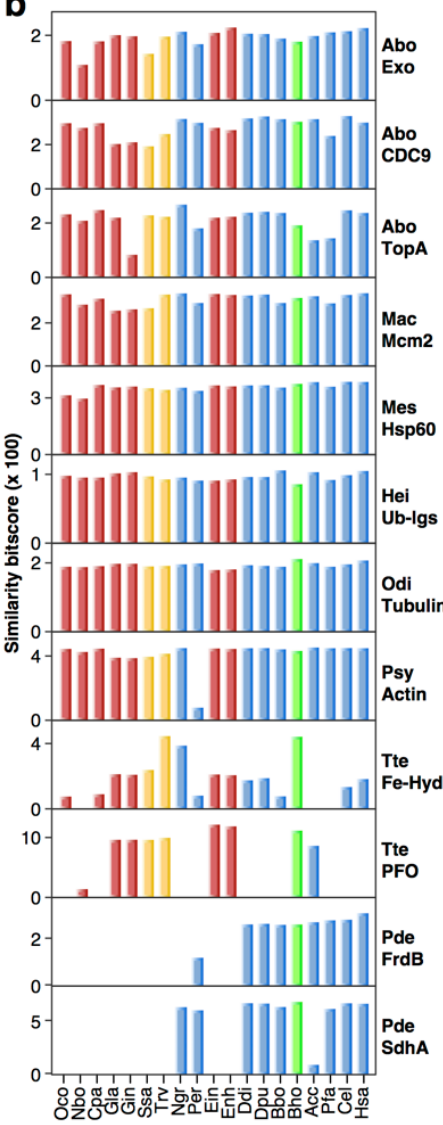

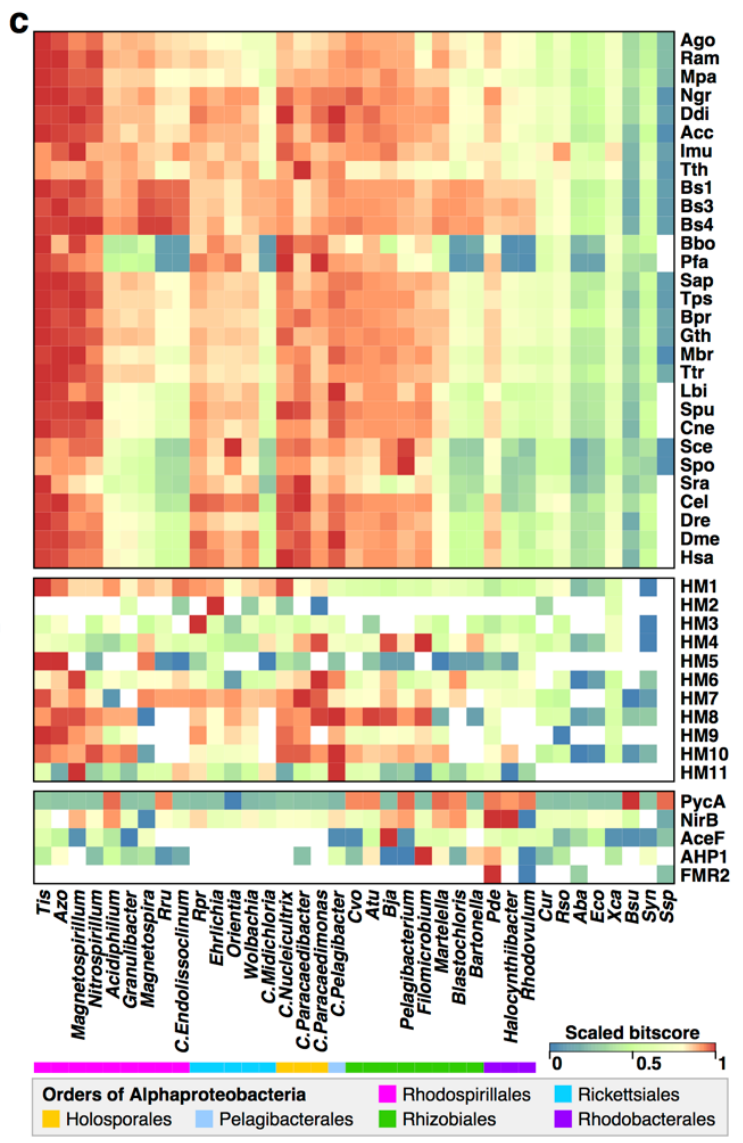

Figure 5. Protein similarities. (a) Similarity bitscores of 'Replication, recombination and repair' enzymes/proteins in Gla or Eae toward their counterparts belonging to the same COG in different archaeons. (b) Similarity bitscores of different eukaryotic proteins (x-axis) toward their prokaryotic counterparts employed as probes (y-axis, on right): Exo, 5' -3' exonuclease; CDC9, ATP-dependent DNA ligase; TopA, DNA topoisomerase IA; Mcm2, DNA replicative helicase MCM subunit Mcm2; Ub-lgs, ubiquitin-protein ligase; Fe-Hyd, iron only hydrogenase; FrdB the $\mathrm{Fe}-\mathrm{S}$ protein subunit, and $\mathrm{SdhA}$ the flavoprotein subunit, of succinate dehydrogenase/fumarate reductase. (c) Top panel: similarity bitscores between bacterial proteomes (x-axis) and total mtDNA-encoded proteins in individual eukaryotes (y-axis). Middle panel: similarity bitscore between each of human mtDNA-encoded proteins HM1-11 (representing NADH dehydrogenase subunits 1-4, 4L and 5, cytochrome b, cytochrome oxidase subunits I-III, and ATP synthase F0 subunit-6 respectively) and its counterpart in different bacterial species. Bottom panel: similarity bitscore between nuclear DNA-encoded eukaryotic proteins PycA, pyruvate carboxylase from Sce; NirB, NAD(P)H-nitrite reductase from Pte; AceF, pyruvate/2-oxoglutarate dehydrogenase complex, dihydrolipoamide acyltransferase (E2) component from Tbr; AHP1, peroxiredoxin from Pfa; or FMR2, fatty acid repression mutant protein from Tbr, and its counterpart in different bacterial species. (See similarity bitscores for Gla, Eae, Trv and Gin in an expansion of part a in Supplementary Figure $\mathrm{S} 1$; bitscore datasets for parts b and c in Supplementary Tables S4-S5; comparison of the probes for Fe-Hyd and PFO from Tte, Ssp and Hth in Supplementary Table S5b; and expanded bitscore datasets relating to mtDNA-encoded proteins in Supplementary Figure S2). 